\title{
A visual quality control scale for clinical arterial spin labeling images
}

\author{
S. M. Fallatah ${ }^{1,2,3}$, F. B. Pizzini $4^{*}$ (D, B. Gomez-Anson ${ }^{5}$, J. Magerkurth', E. De Vita ${ }^{1,2}$, S. Bisdas ${ }^{1,2}$, H. R. Jäger ${ }^{1,2}$, \\ H. J. M. M. Mutsaerts ${ }^{6,7,8+}$ and X. Golay ${ }^{1,2+}$
}

\begin{abstract}
Background: Image-quality assessment is a fundamental step before clinical evaluation of magnetic resonance images. The aim of this study was to introduce a visual scoring system that provides a quality control standard for arterial spin labeling (ASL) and that can be applied to cerebral blood flow (CBF) maps, as well as to ancillary ASL images.

Methods: The proposed image quality control (QC) system had two components: (1) contrast-based QC (cQC), describing the visual contrast between anatomical structures; and (2) artifact-based QC (aQC), evaluating image quality of the CBF map for the presence of common types of artifacts. Three raters evaluated CQC and aQC for 158 quantitative signal targeting with alternating radiofrequency labelling of arterial regions (QUASAR) ASL scans (CBF, T1 relaxation rate, arterial blood volume, and arterial transient time). Spearman correlation coefficient ( $r$ ), intraclass correlation coefficients (ICC), and receiver operating characteristic analysis were used.
\end{abstract}

Results: Intra/inter-rater agreement ranged from moderate to excellent; inter-rater ICC was 0.72 for CQC, 0.60 for aQC, and 0.74 for the combined QC (CQC + aQC). Intra-rater ICC was 0.90 for CQC; 0.80 for aQC, and 0.90 for the combined QC. Strong correlations were found between aQC and CBF maps quality ( $r=0.75)$, and between aQC and CQC $(r=0.70)$. A QC score of 18 was optimal to discriminate between high and low quality clinical scans.

Conclusions: The proposed QC system provided high reproducibility and a reliable threshold for discarding low quality scans. Future research should compare this visual QC system with an automatic QC system.

Keywords: Arterial spin labelling, Magnetic resonance imaging, Perfusion imaging, Quality control

\section{Key points}

- ASL quality control guidelines and standards of acceptance are needed for clinicians

- Visual quality control score is able to select clinically useful scans

- This quality control shows reasonable reproducibility and reliability

- Quality control can be applied to various ASL sequences

\section{Background}

Arterial spin labeling (ASL) is a non-invasive magnetic resonance imaging technique that uses magnetically labelled

\footnotetext{
* Correspondence: francesca.pizzini@aovr.veneto.it

${ }^{\dagger}$ H. J. M. M. Mutsaerts and X. Golay contributed equally to this work.

${ }^{4}$ Neuroradiology, University Hospital of Verona, Piazzale Stefani 1, 37126

Verona, Italy

Full list of author information is available at the end of the article
}

blood water as an endogenous diffusible tracer to quantify cerebral blood flow (CBF) [1]. Because of the tight coupling between brain perfusion and neuronal health, ASL has shown to be an indispensable tool to study brain function in vivo [2-4]. Its non-invasiveness and the lack of an injectable tracer allows longitudinal monitoring of disease progression and treatment efficacy [5].

For the translation of ASL to clinical practice, a wide range of significant developments were performed [6]. Image quality has been improved [7], acquisition times have been reduced [8] and the reliability and reproducibility of ASL perfusion images has been established for multiple centres with different scanners and sequences $[9,10]$. Standardised acquisition methods were agreed upon [1], physiological perfusion confounders were reviewed [11] and standardised image processing methods are developed $[12,13]$. One lacking step for enabling translation of ASL to clinical practice and clinical trials is the development 
and validation of standardised quality control (QC) guidelines [1].

Typically, ASL provides CBF as a single measure of perfusion. However, ASL techniques can be modified to acquire CBF images at multiple post-labelling delays. This offers more information about the labelled bolus and its arrival to the tissue, providing more comprehensive haemodynamic parameters $[14,15]$. One of these techniques is the quantitative signal targeting with alternating radiofrequency labelling of arterial regions (QUASAR) [16]. In addition to CBF maps, QUASAR acquires several other ancillary parametric maps in the same resolution and space as the ASL CBF image $[14,16]$. First, R1 maps are derived from the Look-Locker multiinversion time scheme, representing the longitudinal relaxation rate of the brain tissue. It has contrast similar to a T1-weighted image and, therefore, carries relatively detailed anatomical information. Arterial blood volume (aBV) maps are similar to low-resolution angiography maps, whereas arterial transit time (ATT) maps show the time necessary for the labelled blood to flow from the labelling slab to the vascular compartment of the imaging voxel. ATT maps can be useful to demonstrate the regions of prolonged transit time such as in steno-occlusive diseases [17]. In the normal brain, boundaries between the territories of the anterior, middle, and posterior cerebral arteries (watershed areas) have longer ATT than the core of these perfusion territories, thereby delineating areas prone to borderzone or watershed stroke [17].

This study introduces a visual QC system for the clinical evaluation of ASL perfusion maps. This visual QC system consists of two components: (1) a contrast component that indicates the image contrast between anatomical structures; and (2) an artifact component that scores the presence of image artifacts that degrade image quality, as previously proposed [1]. For a wide range of applicability, this visual QC system was not only developed for CBF maps, but also for other ancillary images that can potentially be acquired. The visual $\mathrm{QC}$ was evaluated in patients with a range of diseases, as well as in healthy volunteers.

\section{Methods}

\section{Participants and study design}

Data for this study originated from several previous studies regarding stroke, multiple sclerosis, QUASAR reproducibility $[9,18-21]$ as well as brain involvement in human immunodeficiency virus (HIV) infection and ECST-2 (Second European Carotid Surgery Trial, http://s489637516.websitehome.co.uk/ECST2/index2.htm); these latter performed at University College of London Hospitals (UCLH) and never published. QUASAR ASL scans of 158 subjects (age $47 \pm$ 17.6 , mean \pm standard deviation; range of $18-98$ years; 81 males) from six Philips 3T scanners from five different centres were included. The mixed study population consisted of 60 healthy volunteers (age $33.7 \pm 8.9$, mean \pm standard deviation; range of 18-65 years; 34 males) from the QUASAR reproducibility study $[1,18,20], 48$ multiple sclerosis patients, 41 stroke patients and nine HIV-positive subjects. All studies were ethically approved by the Research Ethics Committees of studies used in this retrospective analysis (the multiple sclerosis patient study was approved by the Central London Research Ethics Committee number 09/ H0715/45; the European Carotid Surgery Trial 2 (ECST2) by the National Research Ethics Service Committee - East of England, ref.: 11/EE/0347; the HIV patient study by the South East Coast - Surrey Research Ethics Committee 12/ LO/0073; the QUASAR Reproducibility Study by the Singapore National Healthcare Group's Domain Specific Review Board DSRB-A-036). Written informed consent was provided by all subjects.

\section{QUASAR image acquisition and processing}

All imaging was performed on Philips 3T scanners (Achieva, Philips Healthcare, Best, The Netherlands) using the following QUASAR pulse sequence parameters: repetition time $/$ echo time $=4000 / 22.5 \mathrm{~ms} ; 13$ inversion times between $40 \mathrm{~ms}$ and $3640 \mathrm{~ms}$ with an interval of $300 \mathrm{~ms}$, flip angle $35^{\circ}$; field-of-view $240 \times 240 \mathrm{~mm}^{2}$; matrix $64 \times$ 64; seven slices of $6-\mathrm{mm}$ thickness with a 2-mm gap, resulting in a $3.75 \times 3.75 \times 8 \mathrm{~mm}^{3}$ resolution. Label slab thickness was $150 \mathrm{~mm}$; label gap $15 \mathrm{~mm}$; vascular crushers set at $3 \mathrm{~cm} / \mathrm{s}$. All data were processed with QUASAR software $[9,16]$ written in Interactive Data Language (IDL 8.2, ITT Visual Information Solutions, Boulder, CO). Image processing and quantification were performed according to recent consensus [1]. All images were evaluated in native ASL space using ImageJ (National Institutes of Health, Bethesda, MD, USA, v. 1.52e) [22].

\section{Visual QC}

The visual QC score composed of two parts. The contrast-based $\mathrm{QC}(\mathrm{cQC})$ described the visual contrast between anatomical structures and it can be used not only for CBF maps but also for the ancillary parametric maps (R1, aBV, ATT). The scores had a value between 0 and 2, with three items for each CQC maps, with a maximal value of 6 per map (i.e. CBF, R1, aBV, and ATT maps), totalling into 24 for these four cQC maps. The artifact-based QC (aQC) evaluated image quality with respect to common artifacts that can affect ASL CBF maps, and was only used for the CBF maps. Each of the four aQC items (motion, signal drop, distortion, and bright spots, as described below) had a value between 0 and 2, totalling a max of 8 . The total QC score then had a maximum value of $24+8=32$ (Table 1 ). 
Table 1 Items evaluated for the visual quality control of ASL images

Evaluation of CBF maps
Contrast component
Grey matter (0-2)
Grey/white matter differentiation (0-2)
Basal ganglia and thalami (0-2)
Subtotal (0-6)
Artifact component
Motion (0-2)
Signal drop (0-2)
Distortion (0-2)
Bright spots and areas (0-2)
Subtotal (0-8)
Grand total (0-14)

Evaluation of ancillary maps (QUASAR-specific)

Contrast

R1

Grey matter (0-2)

Grey/white matter differentiation (0-2)

Basal ganglia and thalami (0-2)

Subtotal (0-6)

aBV

Anterior cerebral arteries (0-2)

Middle cerebral arteries (0-2)

Posterior cerebral arteries (0-2)

Subtotal (0-6)

ATT

Anterior watershed area (2)

Posterior watershed area (2)

Deep watershed area (2)

Subtotal (0-6)

Grand total (0-18)

A higher score means a better contrast or less artifacts. Range of scores within parentheses. ASL arterial spin labeling, CBF cerebral blood flow, QUASAR quantitative signal targeting with alternating radiofrequency labelling of arterial regions, $R 1$ longitudinal relaxation rate, $a B V$ arterial blood volume, $A T T$ arterial transit time

\section{Contrast-based QC}

For each image, the contrast visibility of three items was assessed (Table 1). Each item was scored from 0 to 2, as follows: clearly visible contrast (score 2), unclear contrast (score 1 ) or no visible contrast (score 0 ). The total score of each map (CBF, R1, aBV, ATT) had a maximum value of 6 , for a maximum achievable cQC score value of 24. Higher scores equate to higher image contrast.

For the $\mathrm{CBF}$ and $\mathrm{R} 1$ maps, the three $\mathrm{CQC}$ items were the cortical grey matter, deep grey matter (i.e. basal ganglia and thalami), and grey matter (GM) to white matter (WM) differentiation. For the aBV maps, these were the contrast visibility of the three major intracerebral arteries: bilateral anterior, middle, and posterior cerebral arteries. These arteries appear as high-intensity vessels on the aBV maps. In the case of low scores, the anatomical images were reviewed to exclude arterial occlusion. For the ATT maps, the three cQC items investigated were the anterior and posterior superficial watershed areas, and the deep watershed area, which lie at the borders of major arterial territories [23]. These watershed areas were evaluated as prolonged ATT times on the ATT maps (Fig. 1).

\section{Artifact-based QC}

Four types of artifacts were assessed on the CBF maps: head motion, signal drop, geometric distortion, and macro-vascular bright spots (Fig. 2). Each item was scored from 0 to 2: no artifacts (score 2), moderate artifacts (score 1) or severe artifacts (score 0 ). The maximum achievable aQC score was 8 , with higher scores equating to fewer image artifacts. Motion artifacts were detected as a hyperintensity rim around the CBF maps (Fig. 2a), which are due to the subtractive nature of ASL.

Signal drop (Fig. 2b) and geometric distortion (Fig. 2c) are the consequence of echo-planar imaging magnetic susceptibility at brain tissue-bone-air interface (susceptibility artifacts). Signal drop occurs frequently in the medial temporal cortex near the mastoid air cells at the base of the skull, as well as in the orbitofrontal cortex near the paranasal sinuses [24]. Some signal drop at the base of the skull is inevitable, and this was only scored when excessive aeration of the sinuses or petrous bone defined as hyperpneumatisation - degraded the image contrast. Geometric distortion was defined as alterations of the outer contour of the image.

Macro-vascular artifacts are recognised as bright spots, due to voxels with a large aBV containing residual labelled blood in the large vessels. A typical example of a macrovascular artifact of the middle cerebral artery is shown on the right in Fig. 2d. Macro-vascular artifacts or bright spots were defined as irregular, asymmetrical, vesselshaped, high-intensity clusters, combined by a surrounding or distal, low-intensity area. Visual or auditory activation can mimic bright foci/spots in the primary visual and auditory cortices [24]. However, these are more often observed as a larger homogeneous area, often bilateral, and not accompanied by a surrounding or distal, low-intensity region [25]. Noise and motion may also present as bright spots [24, 25]. Care was taken to differentiate these causes of bright spots, by the above radiological image features as well as by the knowledge of vascular anatomy, although the latter can differ between subjects. 

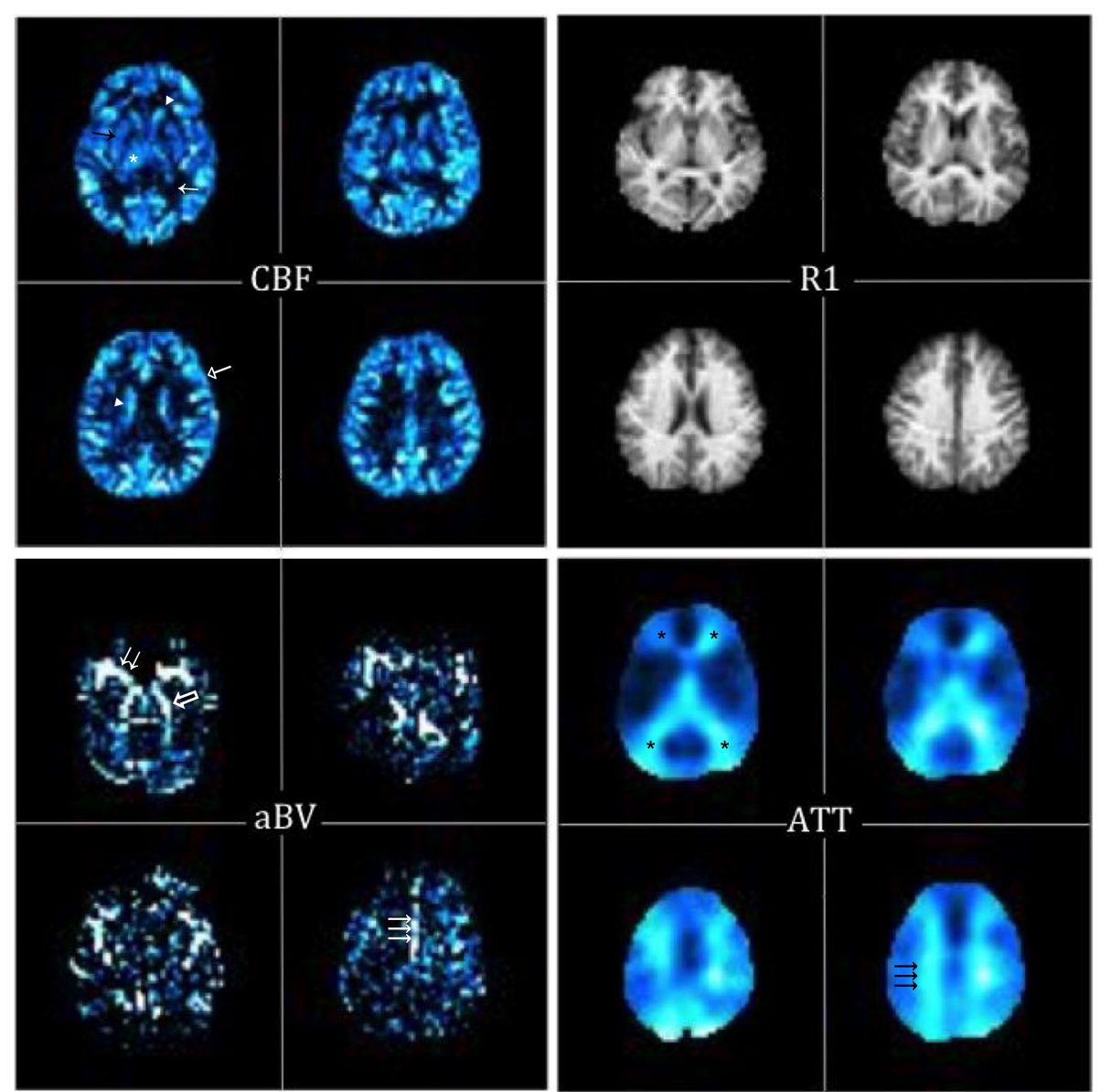

Fig. 1 The cerebral blood flow (CBF) and T1 relaxation rate (R1) maps in the top row show an example of good contrast between the white matter and the cortical and subcortical grey matter: caudate nucleus head and body (arrowheads), thalamus (asterisk), white matter (arrow), grey matter (open-headed arrow). The arterial blood volume (aBV) maps illustrate the larger arterial volume corresponding to the anterior (triple arrow), middle (double arrow) and posterior cerebral (open arrow) arteries. The arterial transit time (ATT) maps show the areas of prolonged ATT in the superficial (asterisks) and deep (triple arrows) watershed areas

\section{Raters}

All maps were independently evaluated within the same time period by three neuroradiologists, S.F. F.P. and B.G. with, respectively 7,10 , and 17 years of experience. Before rating, the raters had a training session to agree on how to score image contrast and artifacts. S.F. performed the rating of all data twice with an interval of 2 months, to assess the intra-rater agreement. Two raters, F.P. and S.B. independently performed an evaluation of the CBF maps to determine whether these were clinically usable or not. A senior neuroradiologist (28 years of experience), R.J. revised any disagreement and provided the final decision as to whether the scans were clinically usable or not. This binary evaluation was used as a reference to define a 'clinically valid' threshold using the receiver operating characteristic (ROC) analysis.

The scoring performed by the rater F.P. was only included in the agreement analysis, being excluded from the ROC analysis because she had participated in the binary classification that was used as a reference.

\section{Statistical analysis}

The Spearman correlation coefficient $(r)$ was used to investigate the relationship between image contrast and artifact scores (cQC and aQC). Intraclass correlation coefficients (ICC) were calculated to determine the levels of inter- and intra-rater agreements. ICC values were interpreted according to the following categorisation: 0 $\leqq$ unusable $<0.2 \leqq$ poor $<0.4 \leqq$ fair $<0.6 \leqq \operatorname{good}<0.8 \leqq$ excellent $\leqq 1.0$ agreement [26, 27].

$\mathrm{CBF}, \mathrm{cQS}, \mathrm{aQS}$, and total visual QC ROC curves were plotted with different thresholds to assess their performance in differentiating clinically usable and unusable ASL scans. Optimal thresholds were defined as those resulting in the maximum area under the curve (AUC). Sensitivity, specificity, positive and negative predictive 


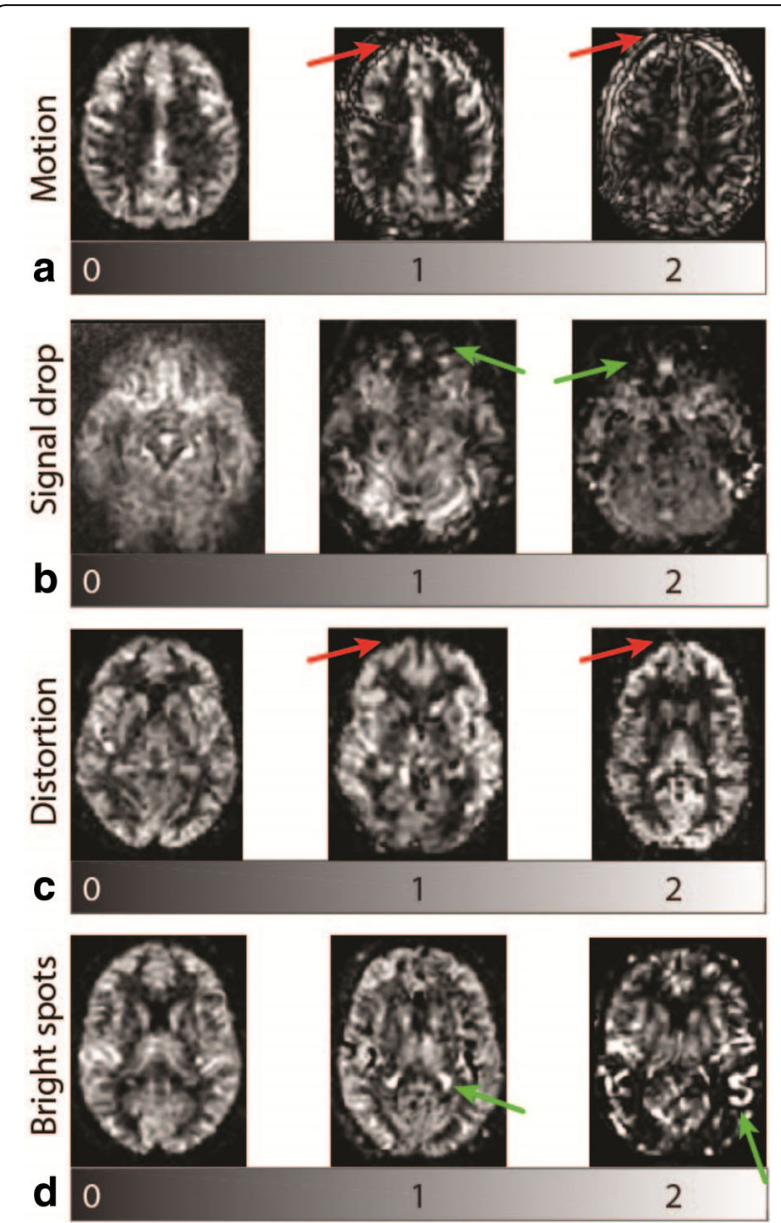

Fig. 2 Cerebral blood flow images containing examples of artifacts: a motion artifacts are observed as pseudo-perfusion hyperintensity rings (red arrows), b signal drop in the inferior orbitofrontal region (green arrows), c geometric anterior-posterior distortion (red arrows), d bright spots depicting macro-vascular artifacts from the posterior cerebral artery behind the thalamus (1) and in the cortex (2, green arrows). Note that the 0 signal drop image is a 3D spiral image, for a better contrast on how the inferior orbitofrontal perfusion should appear without signal drop.

values for differentiating between usable and non-usable ASL scans were also calculated.

\section{Results}

Boxplots in Fig. 3 illustrate the QC scores of the three raters individually for each of the maps. R1 maps consistently showed the highest image contrast (median $=6$ ). The CBF cQC and total QC scores correlated strongly with the aQC $(r=0.75, p<0.001$ and $r=0.70, p<0.001$, respectively, Table 2). Scans with poor $\mathrm{cQC}$ on the aBV maps also scored low on the CBF and ATT maps.

Whereas the total cQC correlated strongly with motion artifacts $(r=0.62, p<0.001)$, it correlated weakly with signal drop and geometric distortion artifacts $(r=$ $0.29, p=0.001$ and $r=0.22, p=0.002$ ), respectively. The macro-vascular bright spots artifacts correlated moderately with the $\mathrm{cQC}(r=0.48, p<0.001)$.

\section{Intra- and inter-rater agreement}

The neuroradiologists agreed in 123 maps and disagreed in 35 maps. The intra-rater agreement was high for $\mathrm{cQC}$ ( $\mathrm{ICC}=0.90)$, for aQC $(\mathrm{ICC}=0.80)$, and for the combined QC (ICC $=0.90$, Table 3). The inter-rater ICC was good for $\mathrm{CQC}(\mathrm{ICC}=0.72)$, for $\mathrm{aQC}(\mathrm{ICC}=0.60)$, and for the combined QC $(\mathrm{ICC}=0.74)$.

Figure 4 shows intra- and inter-rater Bland-Altman plots for the combined QC score. Intra-rater, the 95\% limits of agreement were \pm 5.5 points, which equates to a within-subject coefficient of variation of $29.0 \%$ for a mean score of 19 points. While the mean difference between raters was less than 1.5 points in all three comparisons, the $95 \%$ limits of agreement for inter-rater variation (Fig. $4 \mathrm{a}, \mathrm{b}$, and c) were \pm 8 points in all cases. This equates to a within-subject coefficient of variation of $42.1 \%$ for a mean score of 19 points.

\section{Diagnostic quality}

Table 4 shows the sensitivity and specificity for the detection of clinically usable and non-usable ASL scans, using the cQC for the CBF images only. They were: 79 and 93\%, respectively, for the $\mathrm{CBF} \mathrm{cQC}$ at threshold value of $4 / 6 ; 85$ and $80 \%$ for the total cQC at threshold value of $15 / 24 ; 87$ and $76 \%$ for aQC at threshold value of 4/8; 90 and $80 \%$ for the total QC for threshold value of $18 / 32$ (Table 4 ).

Whereas the CBF cQC had the highest AUC, 0.91 (0.88-0.94 95\% confidence interval (CI)), the aQC had the lowest AUC, 0.88 (0.82-0.90 95\% CI). AUC for the total cQC was $0.89(0.83-0.9195 \% \mathrm{CI})$ and 0.90 for the total QC (0.86-0.93 95\% CI) (Fig. 5).

\section{Discussion}

The main finding of this study is that the developed visual QC score system showed robustness within and between raters. The neuroradiologists felt this to be a helpful and easy-to-use rating system that provides an image quality indication before using ASL for any clinical or research assessment.

We provide several threshold scores as guidelines to determine whether or not the ASL image is of diagnostic quality. For the combined QC as used for QUASAR, a threshold of 18 seemed to be a robust choice. The fact that the CBF cQC score had a slightly higher performance can be explained by the fact that the reference for diagnostic usability was derived mainly from the CBF maps. Hence, for clinical single post-labelling delay pseudo-continuous ASL sequences, a CBF cQC threshold of 4 can be used as a diagnostic quality guideline. A CBF cQC score of 4 had the highest AUC (90\%), and 


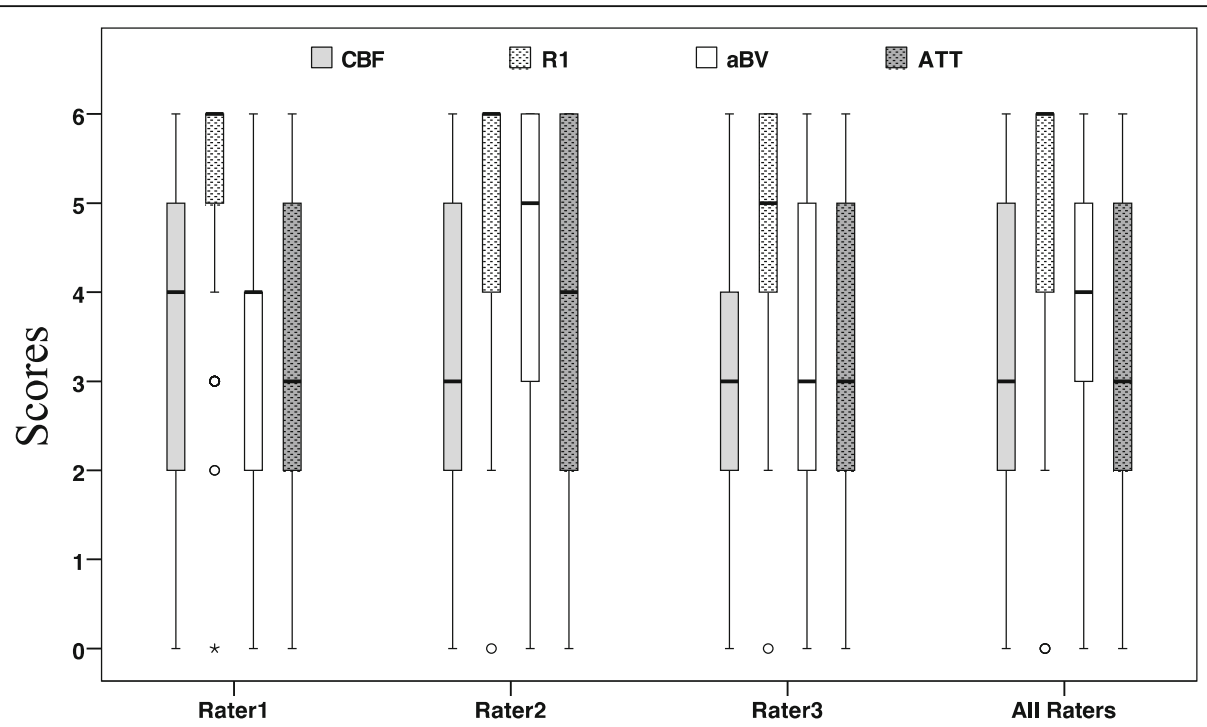

Fig. 3 Visibility scores for each of the individual raters and all the data from the three raters. The T1 relaxation rate (R1) map has the highest score, median score of 6 averaged for all raters

this threshold predicted clinical usability with specificity and sensitivity of 90 and $75 \%$, respectively.

The high correlation between $\mathrm{cQC}$ and $\mathrm{aQC}$ may not be surprising, as image artifacts can obscure perfusion contrast. Interestingly, the motion aQC had the highest correlation with the $\mathrm{CQC}$. This fits with a previous simulation study [28], which showed that motion had a smoothing effect on the contrast between GM and WM CBF across the brain. Motion artifacts are known to have a high impact on ASL image quality, due to the subtractive nature of the technique [1]. This is particularly emphasised for ASL sequences without background suppression, as was the case for QUASAR in this study.

The lack of a correlation between signal drop aQC and $\mathrm{cQC}$ could be explained by the fact that this susceptibility

Table 2 Spearman's correlation coefficient between the first (QC) and second (items) columns for raters individually (columns 3,4 and 5) and for all raters in the last column

\begin{tabular}{llllll}
\hline QC & Item & Rater 1 & Rater 2 & Rater 3 & All \\
\hline cQC & CBF & 0.85 & 0.78 & 0.60 & 0.75 \\
cQC & R1 & 0.59 & 0.46 & 0.56 & 0.48 \\
cQC & aBV & 0.57 & 0.40 & 0.36 & 0.42 \\
cQC & ATT & 0.48 & 0.56 & 0.36 & 0.46 \\
QC & Total cQC & 0.80 & 0.74 & 0.60 & 0.70 \\
aQC & Motion & 0.70 & 0.56 & 0.65 & 0.62 \\
aQC & Signal dropout & 0.24 & 0.28 & 0.44 & 0.29 \\
aQC & Distortion & 0.21 & 0.25 & 0.22 & 0.22 \\
aQC & Bright spots & 0.58 & 0.63 & 0.26 & 0.48 \\
\hline
\end{tabular}

$a B V$ arterial blood volume $A T T$ arterial transit time, $C B F$ cerebral blood flow, $a Q C$ artifact-based quality control, $C Q C$ contrast-based quality control, $R 1 \mathrm{~T} 1$ relaxation rate artifact is inevitable in several clinical magnetic resonance imaging (MRI) acquisitions, and are well-known and tolerated by radiologists. While its extent may vary largely between subjects because of difference normal variants of sinuses and air cavities, the locations and appearance of these artifacts are well-known. This led also to the pragmatic choice of accepting a slight signal dropout near the base of the skull as clinically usable, and normal. The other result of susceptibility artifacts - geometric distortion - also did not correlate with cQC. Geometric distortion is relatively mild in ASL compared to other advanced techniques, such as functional MRI and diffusion tensor imaging, as the readout length is typically shorter than in the latter techniques [29]. Moreover, this distortion does not necessarily change image contrast.

The raters had a good visual training regarding the main physiological and vascular anatomy variations expected in patients, so they did not report any particular difficulties to differentiate between them and the noiseor motion-related causes of bright spots. There was a moderate positive correlation between the bright spot $\mathrm{aQC}$ score and the general cQC $(r=0.48, p=0.001)$.

Notably, although the primary goal of our study was to provide guidelines for radiologists to accept or discard

Table 3 Intra- and inter-rater intraclass correlation coefficient(ICC) values

\begin{tabular}{llllllll}
\hline ICC & CBF & R1 & aBV & ATT & CQC & aQC & Total QC \\
\hline Intra-rater & 0.87 & 0.84 & 0.77 & 0.86 & 0.90 & 0.80 & 0.90 \\
Inter-rater & 0.70 & 0.50 & 0.45 & 0.63 & 0.72 & 0.60 & 0.74 \\
\hline
\end{tabular}

$a B V$ arterial blood volume, $a Q C$ artifact-based quality control, $A T T$ arterial transit time, $C B F$ cerebral blood flow, $C Q C$ contrast-based quality control, $R 1 \mathrm{~T} 1$ relaxation rate 

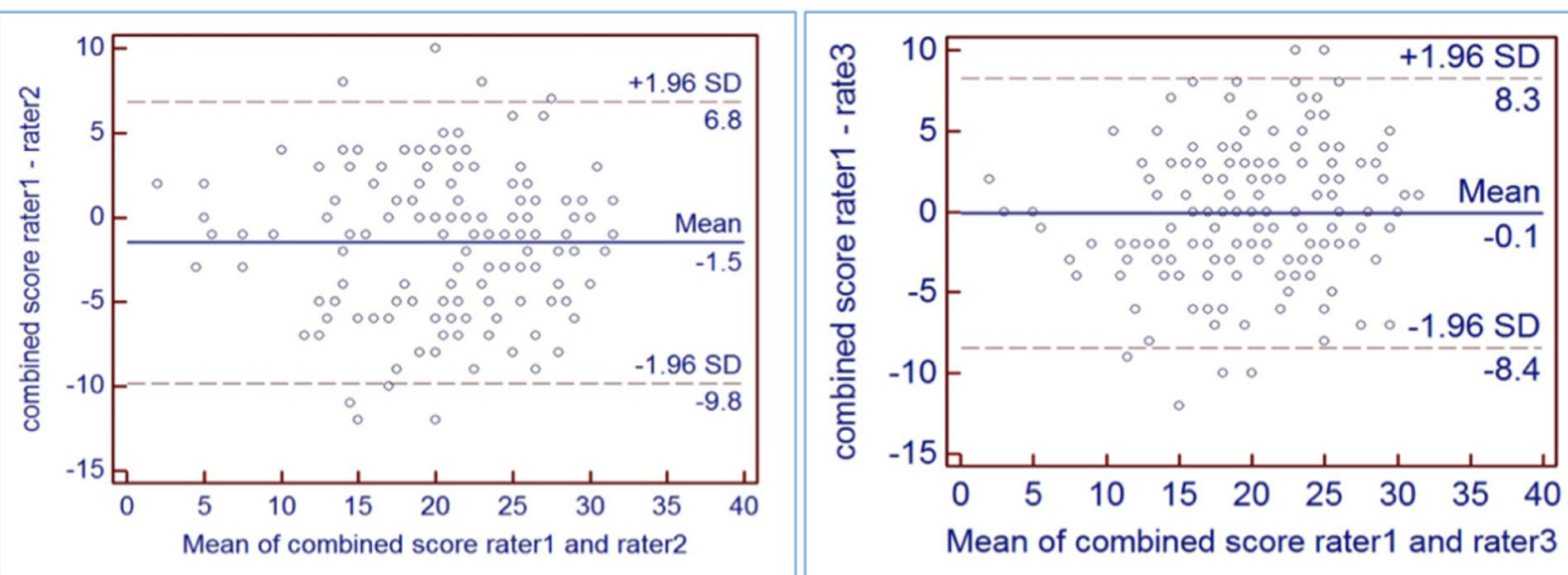

Mean of combined score rater 1 and rater3
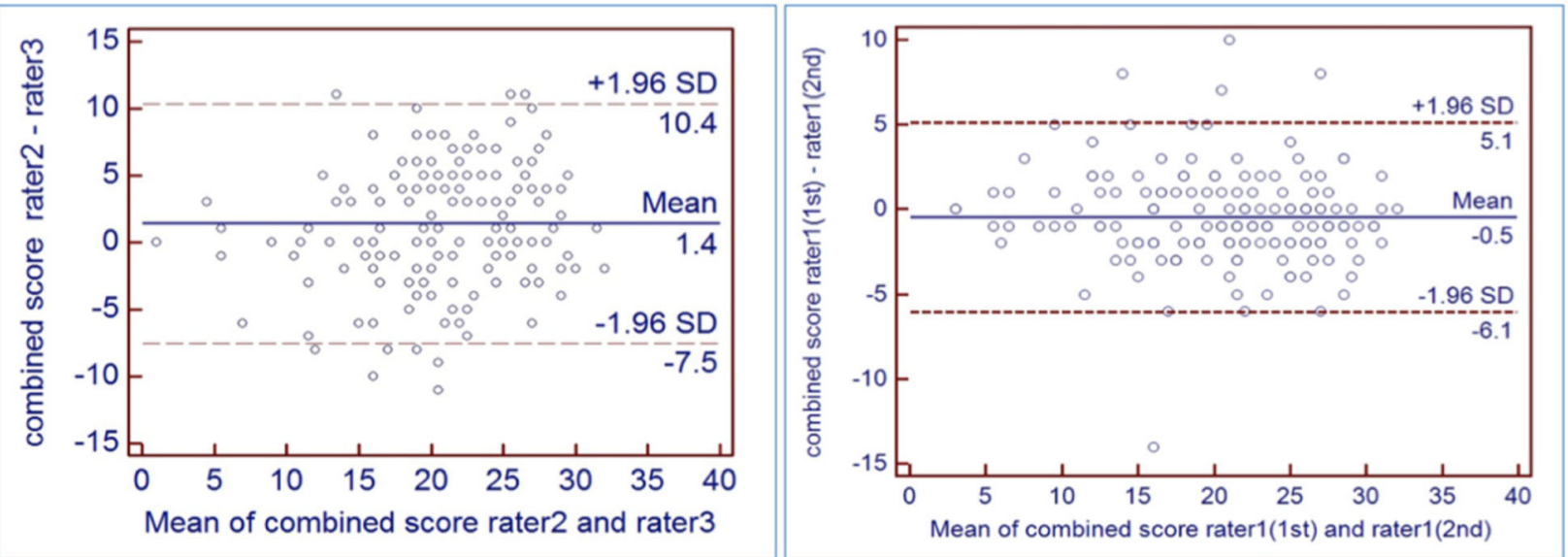

Fig. 4 Bland-Altman plots illustrate inter (1-3) and intra-rater (4) variation of the combined score measurements. Red dashed lines are $95 \% \mathrm{Cl}$ of limits of repeatability, and the blue line is the mean difference.

an individual ASL scan based on visual QC, this scoring system can also be used as a teaching tool for neuroradiologists that are not familiar to ASL, to differentiate artifacts from perfusion changes.

The visual differentiation between normal anatomical variants and acquisition artifacts can be difficult. We acknowledge several conditions where pathology is known to simulate acquisition artifacts. A frequently occurring example is the signal drop due to a pathologically prolonged bolus arrival time. A more rare example is that the bright areas are explained by a pathological

Table 4 Performance of CBF, QC, CQC, and aQC

\begin{tabular}{lllll}
\hline & CBF CQC & Total QC & Total cQC & aQC \\
\hline Score & $4 / 6$ & $19 / 32$ & $15 / 24$ & $4 / 8$ \\
Sensitivity (\%) & 75 & 87 & 88 & 90 \\
Specificity (\%) & 90 & 73 & 73 & 68 \\
PPV (\%) & 91 & 84 & 83 & 81 \\
NPV (\%) & 70 & 78 & 79 & 82 \\
\hline
\end{tabular}

$a Q C$ artifact-based quality control, $C Q C$ contrast-based quality control, $C B F$ cerebral blood flow, NPV negative predictive value, PPV positive predictive value, $Q C$ quality control

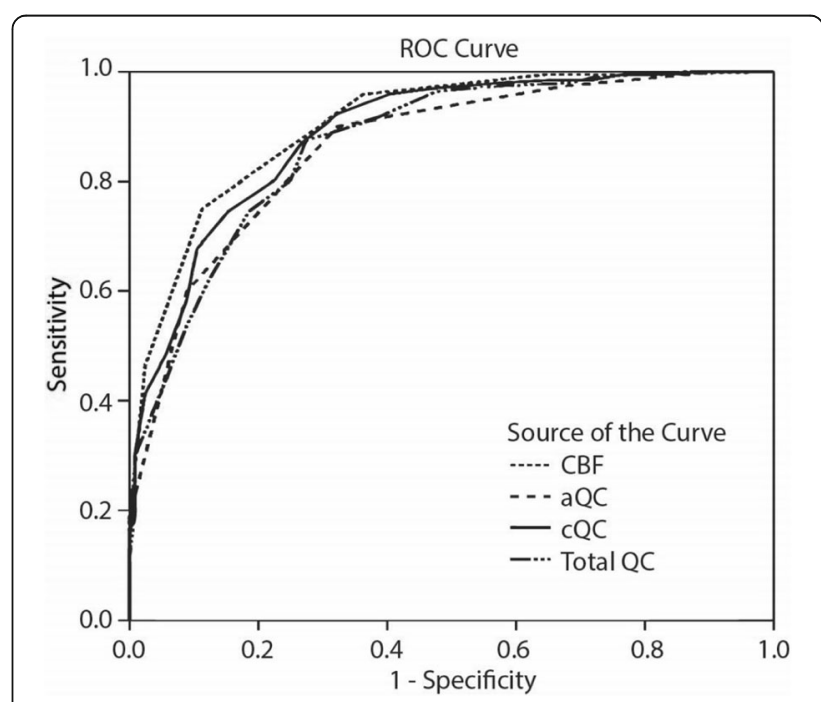

Fig. 5 Receiver-operator characteristic (ROC) curves of cerebral blood flow (CBF) CQC, contrast-based quality control (CQC), artifactbased QC ( $\mathrm{aQC})$ and total QC. The areas under the curve for these 4 parameters are, respectively: $0.90,0.89,0.87$, and 0.86 
arteriovenous shunt [30]. In the first case, the typical anatomical vessel distribution of the signal drop areas can suggest the presence of an unknown extracranial vessel stenosis, while in the second one, the serpiginous shape of the bright areas, the bright signal in the main drainage veins and sinuses can indicate the presence of an arteriovenous shunt. The corresponding conventional anatomical MRI images can show a cluster of enlarged vessels in the latter case, due to an arteriovenous malformation, or a thin or absent flow void in T2- and T2*-weighted gradient-echo sequences of the intracranial portion of vessels, in case of a stenosis or occlusion. In doubtful cases, we suggest a pragmatic approach of taking into account the corresponding anatomical images and comparing them to the ASL maps to avoid treating physiological changes as image artifacts.

This study suffers from several potential limitations. Both the scoring system and the diagnostic usability which was used as a reference, are subjective. Future work may compare this clinical visual scoring system with existing parametric scoring systems, which may be more objective [31-34]. However, these parameters are sensitive to both instrumental and (patho-)physiological changes, making them less reliable for clinical use. This is especially important in the case of ASL, because its signal-to-noise ratio is directly related to $\mathrm{CBF}$ and other physiological alterations, such as haematocrit or oxygenation changes, hence clinical knowledge is required to distinguish instrumental artifacts from artifacts that are disease-related and inevitable [35]. Future work should investigate the combined performance of a visual and parametric QC in clinical applications of ASL.

On the other hand, the fact that image contrast and the presence of motion and vascular artifacts are disease-related in ASL, is a potential limitation to our visual QC as well. To this end, we included both ASL scans from patients and healthy controls. Although pathology could also affect our scoring, these changes are often focal compared to a more widespread acquisitionrelated quality decrease. Nevertheless, there remain cases where the differentiation between acquisition- or pathology-related quality decreases are difficult to assess, e.g. the GM-to-WM contrast loss in labelling asymmetry could appear the same as in a unilateral infarct. To differentiate these, we recommend to evaluate the anatomical MR images and perform a double comparison between them, as suggested above.

We found that the scan quality in patients appeared visually inferior to that of healthy controls, which was correlated to motion and a loss of image contrast, and vascular artifacts. Nevertheless, the majority of patient scans were considered clinically usable in the binary classification. However, it should be noticed that the poorer $\mathrm{CQC}$ and $\mathrm{aQC}$ scores for
ASL scans from patients need to be accepted to a certain degree.

Another limitation to this study is that we only tested our scoring system in QUASAR scans with a two-dimensional (2D) echo-planar readout, which are not the type of scans recommended by the white paper [1]. Although image contrast and artifacts are expected to appear similar on other ASL sequences with a $2 \mathrm{D}$ echoplanar readout, they may differ with three-dimensional (3D) ASL readouts. The main difference is a lower effective spatial resolution for the 3D readouts that are used in ASL: 3D spiral and 3D gradient and spin-echo, mostly because of their wider acquisition point-spread function [36, 37]. These sequences still have a lower GM-to-WM contrast, less visibility and higher sensitivity for motion artifacts. Furthermore, these 3D sequences have a lower degree of geometric distortion and signal dropout, especially the 3D spiral sequence.

In conclusion, the proposed scoring system provides a robust visual quality control for QUASAR ASL images. The scoring system has demonstrated the ability to select clinically useful scans and shows reasonable reproducibility and reliability. Our results encourage future efforts to expand on our quality control guidelines for this growing technique.

\section{Abbreviations \\ 2D: Two-dimensional; 3D: Three-dimensional; aBV: Arterial blood volume; aQC: Artifact-based quality control; ASL: arterial spin labeling; ATT: Arteria transit time; AUC: Area under the curve; CBF: Cerebral blood flow; CQC: Contrast-based quality control; GM: Grey matter; HIV: Human immunodeficiency virus; ICC: Intraclass correlation coefficients; QC: Quality control; QUASAR: Quantitative signal targeting with alternating radiofrequency labelling of arterial regions; $\mathrm{R} 1$ : T1 relaxation rate; ROC: Receiver operating characteristic; WM: White matter}

Availability of data and materials

The datasets generated and analysed during the current study are not publicly available because some of them originated from multicentre unpublished

studies, but are available from the corresponding author on reasonable request.

Funding

This research received a proportion of funding from the UK Department of Health's National Institute for Health Research Biomedical Research Centres Funding scheme. European Cooperation in Science and Technology, BMBS COST Action BM1 103; SF received financial support from the Saudi Arabian Government. HM is supported by Amsterdam Neuroscience funding

\section{Authors' contributions}

SF organised and analysed data and drafted the manuscript. SF, FP, BG, and SB reviewed and rated the data. SF, FP, and HM prepared the figures. XG designed the study and reviewed the manuscript with RJ and HM. All authors critically reviewed the manuscript and approved the final manuscript as submitted and agree to be accountable for all aspects of the work.

Ethics approval and consent to participate

The data used in this study were approved by the Research Ethics Committees from the various original studies used in this retrospective analysis and written informed consent was provided by all subjects.

Consent for publication

Consent to publish obtained in the written informed consent forms. 


\section{Competing interests}

XG declares that he is CEO, holds shares, and receives payment from Gold Standard Phantoms Limited. All the other authors declare that they have no competing interests.

\section{Publisher's Note}

Springer Nature remains neutral with regard to jurisdictional claims in published maps and institutional affiliations.

\section{Author details}

'Department of Brain Repair and Rehabilitation, UCL Institute of Neurology, London, UK. ${ }^{2}$ The National Hospital for Neurology and Neurosurgery, London, UK. ${ }^{3}$ Radiology Department, King Abdualaziz Medical City, Riyadh, Saudi Arabia. ${ }^{4}$ Neuroradiology, University Hospital of Verona, Piazzale Stefani 1, 37126 Verona, Italy. ${ }^{5}$ Unitat Neuroradiologia, Hospital de la Santa Creu i Sant Pau, IIB-Sant Pau, Universitat Autonoma, Barcelona, Spain. ${ }^{6}$ Radiology Department, Academic Medical Center, Amsterdam, The Netherlands. Radiology Department, Vrije Universiteit University Medical Center, Amsterdam, The Netherlands. ${ }^{8}$ Radiology Department, University Medical Center Utrecht, Utrecht, The Netherlands.

\section{Received: 22 June 2018 Accepted: 17 October 2018}

\section{Published online: 19 December 2018}

\section{References}

1. Alsop DC, Detre JA, Golay X et al (2014) Recommended implementation of arterial spin-labeled perfusion MRI for clinical applications: a consensus of the ISMRM perfusion study group and the European Consortium for ASL in dementia. Magn Reson Med 73:102-116

2. Borogovac A, Asllani I (2012) Arterial spin labeling (ASL) fMRl: advantages, theoretical constrains and experimental challenges in neurosciences. Int J Biomed Imaging 2012:818456

3. Hendrikse J, Petersen ET, Golay X (2012) Vascular disorders: insights from arterial spin labeling. Neuroimaging Clin N Am 22:259-269

4. Alsop DC, Dai W, Grossman M, Detre JA (2010) Arterial spin labeling blood flow MRI: its role in the early characterization of Alzheimer's disease. J Alzheimers Dis 20:871-880

5. Wang DJ, Chen Y, Fernández-Seara MA, Detre JA (2011) Potentials and challenges for arterial spin labeling in pharmacological magnetic resonance imaging. J Pharmacol Exp Ther 337:359-366

6. Detre JA, Rao H, Wang DJ, Chen YF, Wang Z (2012) Applications of arterial spin labeled MRI in the brain. J Magn Reson Imaging 35:1026-1037

7. Alsop DC, Detre JA (1996) Reduced transit-time sensitivity in noninvasive magnetic resonance imaging of human cerebral blood flow. J Cereb Blood Flow Metab 16:1236-1249

8. Fernández-Seara M, Edlow BL, Hoang A, Wang J, Feinberg DA, Detre JA (2008) Minimizing acquisition time of arterial spin labeling at 3T. Magn Reson Med 59:1467-1471

9. Petersen ET, Mouridsen K, Golay X (2010) The QUASAR reproducibility study, Part II: results from a multi-center Arterial Spin Labeling test-retest study. Neuroimage 49:104-113

10. Mutsaerts HJ, van Osch MJ Zelaya FO et al (2015) Multi-vendor reliability of arterial spin labeling perfusion MRI using a near-identical sequence: Implications for multi-center studies. Neuroimage 113:143-152

11. Clement P, Mutsaerts HJ, Václavů L et al (2018) Variability of physiological brain perfusion in healthy subjects - A systematic review of modifiers. Considerations for multi-center ASL studies. J Cereb Blood Flow Metab 38: 1418-1437

12. Zhao MY, Mezue M, Segerdahl AR et al (2017) A systematic study of the sensitivity of partial volume correction methods for the quantification of perfusion from pseudo-continuous arterial spin labeling MRI. Neuroimage 162:384-397

13. Mutsaerts H, Petr J, Lysvik E et al (2017) ExploreASL: image processing toolbox for multi-center arterial spin labeling population analyses. Presentation number 733 at Software Exhibit Area, Abstract ID $=1180$ European Society For Magnetic Resonance in Medicine and Biology, Barcelona

14. Heijtel DF, Petersen ET, Mutsaerts HJ et al (2016) Quantitative agreement between $\left[{ }^{15} \mathrm{O}\right] \mathrm{H}_{2} \mathrm{O}$ PET and model free QUASAR MRI-derived cerebral blood flow and arterial blood volume. NMR Biomed 29:519-526
15. Mak HK, Chan Q, Zhang Z et al (2012) Quantitative assessment of cerebral hemodynamic parameters by QUASAR arterial spin labeling in Alzheimer's disease and cognitively normal Elderly adults at 3-tesla. J Alzheimers Dis 31:33-44

16. Petersen ET, Lim T, Golay X (2006) Model-free arterial spin labeling quantification approach for perfusion MRI. Magn Reson Med 55:219-232

17. Donahue MJ, Achten E, Cogswell PM et al (2018) Consensus statement on current and emerging methods for the diagnosis and evaluation of cerebrovascular disease. J Cereb Blood Flow Metab 38:1391-1417

18. Paling D, Thade Petersen E, Tozer DJ et al (2014) Cerebral arterial bolus arrival time is prolonged in multiple sclerosis and associated with disability. I Cereb Blood Flow Metab 34:34-42

19. Godi C, Sokolska M, Kennedy F, Fallatah S, Golay X, Jager HR (2015) Noninvasive quantification of brain perfusion and cerebral haemodynamics with quasar arterial spin labeling (ASL) in patients with carotid atherosclerotic stenosis. Eur Soc Magn Reson Med Biol, Edinburgh, p 469

20. Chng SM, Petersen ET, Zimine I, Sitoh YY, Lim CC, Golay X (2008) Territorial arterial spin labeling in the assessment of collateral circulation: comparison with digital subtraction angiography. Stroke 39:3248-3254.

21. Hendrikse J, Petersen ET, Chng SM, Venketasubramanian N, Golay X (2010) Distribution of cerebral blood flow in the nucleus caudatus, nucleus lentiformis, and thalamus: a study of territorial arterial spin-labeling MR imaging. Radiology 254:867-875

22. Schneider CA, Rasband WS, Eliceiri KW (2012) NIH Image to ImageJ : 25 years of image analysis. Nat Methods 9:671-675

23. Hendrikse J, Petersen ET van Laar PJ, Golay X (2008) Cerebral border zone between distal end branches of intracranial arteries: MR imaging. Radiology 246:572-580

24. Deibler AR, Pollock JM, Kraft RA, Tan H, Burdette JH, Maldjian JA (2008) Arterial spin-labeling in routine clinical practice, part 1: technique and artifacts. AJNR Am J Neuroradiol 29:1228-1234

25. Grade M, Hernandez Tamames JA, Pizzini FB, Achten E, Golay X, Smits M (2015) A neuroradiologist's guide to arterial spin labeling MRI in clinical practice. Neuroradiology 57:1181-1202

26. Rothwell PM (2000) Analysis of agreement between measurements of continuous variables: General principles and lessons from studies of imaging of carotid stenosis. J Neurol 247:825-834

27. Koo TK, Li MY (2016) A guideline of selecting and reporting intraclass correlation coefficients for reliability Research. J Chiropr Med 15:155-163

28. Petr J, Mutsaerts H, de Vita E, et al (2016) Influence of realignment-induced interpolation errors on the estimation of cerebral blood flow using arterial spin labeling fMRI. Magn Reson Mater Phy 29(Suppl 1): 1. https://doi.org/10.1007/ s10334-016-0568-x

29. Jezzard P, Clare S (1999) Sources of distortion in functional MRI data. Hum Brain Mapp 8:80-85

30. Amukotuwa SA, Marks MP, Zaharchuk G, Calamante F, Bammer R, Fischbein N (2018) Arterial spin-labeling improves detection of intracranial dural arteriovenous fistulas with MRI. AJNR Am J Neuroradiol 39:669-677

31. Mutsaerts HJ, Petr J, Václavů L et al (2017) The spatial coefficient of variation in arterial spin labeling cerebral blood flow images. J Cereb Blood Flow Metab 37:3184-3192

32. Dolui S, Wang Z, Shinohara RT, Wolk DA, Detre JA (2016) Structural correlation-based outlier rejection (SCORE) algorithm for arterial spin labeling Time Series. J Magn Reson Imaging 45:1786-1797

33. Shirzadi Z, Crane DE, Robertson AD et al (2015) Automated removal of spurious intermediate cerebral blood flow volumes improves image quality among older patients: a clinical arterial spin labeling investigation. J Magn Reson Imaging 42:1377-1385

34. Mortamet B, Bernstein M, Jack CR Jr et al (2009) Automatic quality assessment in structural brain magnetic resonance imaging. Magn Reson Med 62:365-372

35. Hales PW, Kawadler JM, Aylett SE, Kirkham FJ, Clark CA (2014) Arterial spin labeling characterization of cerebral perfusion during normal maturation from late childhood into adulthood: normal 'reference range' values and their use in clinical studies. J Cereb Blood Flow Metab 34:776-784

36. Vidorreta M, Wang Z, Rodríguez I Pastor MA, Detre JA, Fernández-Seara MA (2013) Comparison of 2D and 3D single-shot ASL perfusion fMRI sequences. Neuroimage 66:662-671

37. Mutsaerts HJMM, Petr J, Thomas DL et al (2018) Comparison of arterial spin labeling registration strategies in the multi-center GENetic frontotemporal dementia initiative (GENFI). J Magn Reson Imaging 47:131-140 